\title{
MODELOS DE DISTRIBUIÇÃO DIAMÉTRICA PARA POVOAMENTOS DESBASTADOS DE EUCALIPTO UTILIZANDO A FUNÇÃO HIPERBÓLICA ${ }^{1}$
}

\author{
Daniel Henrique Breda Binoti², Mayra Luiza Marques da Silva Binoti ${ }^{3}$, Helio Garcia Leite ${ }^{4}$, Silvana Lages \\ Ribeiro Garcia ${ }^{5}$ Jovane Pereira da Cruz $^{6}$
}

\begin{abstract}
RESUMO - Os objetivos deste estudo foram ajustar, empregar e propor modelos de distribuição diamétrica com diferentes formas da função hiperbólica para a projeção da distribuição diamétrica de povoamentos desbastados. Foram utilizados dados de um experimento sobre desbastes, instalado em três povoamentos de um híbrido Eucalyptus grandis x Eucalyptus urophylla, localizado na região Nordeste do Estado da Bahia. As parcelas permanentes foram mensuradas aos $27,40,50,58,61,76,87,101$ e 112, 125, 135, 147 e 160 meses, com um desbaste realizado aos 58 meses e um segundo aos 130 meses. A utilização da função com dois parâmetros apresentou melhores resultados de raiz quadrada do erro médio (RMSE), bias e bias\%.
\end{abstract}

Palavras-chave: Função hiperbólica truncada, Desbaste e Multiprodutos.

\section{DIAMETRIC DISTRIBUTION MODEL THINNED EUCALYPT STANDS FOR USING A HYPERBOLIC FUNCTION}

\begin{abstract}
The objectives of this study were to adjust, to employ and to propose diameter distribution models with different hyperbolic function shapes for the projection of the diameter distribution of thinned stands. It was used data from an experiment on thinning set up in three stands of an Eucalyptus grandis $\times$ Eucalyptus urophylla hybrid, located in the northeastern Bahia state. The permanent plots were measured at $27,40,50,58,61,76,87,101$ and 112, 125, 135, 147 and 160 months, with a thinning carried out at 58 months of age and a second thinning conducted at 130 months of age. The two-parameter function showed the best results of root mean square error (RMSE), bias and bias\%.
\end{abstract}

Keywords: Truncated hyperbolic function, Thinning and Multiproducts.

\section{INTRODUÇÃO}

Os povoamentos florestais destinados à produção de multiprodutos são caracterizados pelo longo período de tempo entre os investimentos iniciais e os recebimentos futuros em função da necessidade de árvores de maiores dimensões, que na maioria das vezes exige a aplicação de desbastes e desrama artificial. Nesse sentido, a predição ou projeção do crescimento e da produção dos povoamentos florestais em nível de árvore ou de classe de diâmetro é essencial para a definição da quantidade dos diversos produtos que serão produzidos pelo povoamento (GUIMARÃES, 1994; DEMOLINARI et al., 2007).

Modelos de distribuição diamétrica (MDD) estimam o crescimento e a produção por classe de diâmetro, permitindo a análise econômica de multiprodutos e a simulação de desbastes (BURKHART et al., 1981; CLUTTER et al., 1983; NOGUEIRA et al., 2005). A eficiência desses modelos é influenciada pelas características da função de densidade probabilidade

\footnotetext{
${ }^{1}$ Recebido em 03.09.2010 e aceito para publicação em 02.05.2011.

${ }^{2}$ Programa de Pós-Graduação em Ciência Florestal pela Universidade Federal de Viçosa, UFV, MG. E-mail: <danielbinoti@yahoo.com.br>. ${ }^{3}$ Departamento de Engenharia Florestal, Universidade Federal dos Vales do Jequitinhonha e Mucuri, UFVJM , MG. E-mail: $<$ mayra_ufv@yahoo.com.br>.

${ }^{4}$ Departamento de Engenharia Florestal, Universidade Federal de Viçosa, UFV, MG. E-mail: <hgleite@gmail.com>.

${ }^{5}$ Faculdade de Viçosa, FDV, Brasil. E-mail: < garciaslr@yahoo.com.br>.

${ }^{6}$ Bahia Pulp. E-mail: <jovane_cruz@bahiapulp.com>.
} 
utilizada. A capacidade de correlação dos parâmetros da função com características do povoamento é fator limitante para a construção das equações que compõem o MDD.

Estudos sobre modelagem da distribuição diamétrica destacam o emprego da função hiperbólica (GUIMARÃES, 2002; CAMPOS; LEITE, 2009; LEITE et al., 2010). Essa função pode, em sua formulação completa, ser descrita como:

$$
f(x)=\left\{\frac{\gamma}{\beta}\left(\frac{x-\alpha}{\beta}\right)^{(\gamma-1)}\left(1-\tanh \left(\left(\frac{x-\alpha}{\beta}\right)^{\gamma}\right)^{2}\right)\right.
$$

em que $\alpha$ é o parâmetro de locação, $\beta$ o parâmetro de escala $(\beta>0), \gamma$ o parâmetro de forma $(\gamma>0)$ e $x$ o centro de classe de diâmetro $(x>0)$.

Estudos de modelagem utilizando a função hiperbólica para povoamentos submetidos a desbastes ainda são escassos. Por isso, objetivou-se neste estudo ajustar e empregar modelos de distribuição diamétrica com diferentes formas da função hiperbólica para a projeção da distribuição diamétrica desse tipo de povoamento.

\section{MATERIAL E MÉTODOS}

\subsection{Dados}

Foram utilizados dados de um experimento sobre desbastes, instalado em três povoamentos de um híbrido Eucalyptus grandis x Eucalyptus urophylla (Rio Claro), em uma empresa florestal localizada na região Nordeste do Estado da Bahia, Brasil. O experimento foi instalado em três instalações, compostos por quatro tratamentos e seis blocos, sendo dois destes em cada instalação. Cada bloco continha duas repetições, totalizando 48 parcelas, com área útil de $2.600 \mathrm{~m}^{2}$, sendo o espaçamento inicial entre as árvores de 3,0 x 3,0 m. Os tratamentos aplicados corresponderam a 20\%, 35\% e 50\% de área basal removida em cada desbaste, eliminando-se os piores indivíduos em função do crescimento volumétrico.

As parcelas permanentes foram mensuradas aos 27, 40, 50, 58, 61, 76, 87, 101 e 112, 125, 135, 147 e 160 meses, com um desbaste realizado aos 58 meses e um segundo aos 130 meses. Em cada medição, foram mensurados os diâmetros a 1,3 de altura (dap), de todas as árvores da parcela.

\subsection{Descrição das Funções}

A $f d p$ da função hiperbólica de três parâmetros (3P) pode ser descrita como:

$$
f(x)=\left\{\frac{\gamma}{\beta}\left(\frac{x-\alpha}{\beta}\right)^{(\gamma-1)}\left(1-\tanh \left(\left(\frac{x-\alpha}{\beta}\right)^{\gamma}\right)^{2}\right)\right.
$$

em que $\alpha$ é o parâmetro de locação, $\beta$ o parâmetro de escala $(\beta>0), \gamma$ o parâmetro de forma $(\gamma>0)$ e $x$ o centro de classe de diâmetro $(x>0)$. A supressão do parâmetro de locação da função caracteriza a função hiperbólica de dois parâmetros (2P), conforme apresentado na equação 2 .

$$
f(x)=\left\{\frac{\gamma}{\beta}\left(\frac{x}{\beta}\right)^{(\gamma-1)}\left(1-\tanh \left(\left(\frac{x}{\beta}\right)^{\gamma}\right)^{2}\right)\right.
$$

O parâmetro de locação pode ser substituído pelo diâmetro mínimo do povoamento ( $\alpha=d \min$ ), sendo ajustado da seguinte forma:

$f(x)=\left\{\frac{\gamma}{\beta}\left(\frac{x-d \min }{\beta}\right)^{(\gamma-1)}\left(1-\tanh \left(\left(\frac{x-d \min }{\beta}\right)^{\gamma}\right)^{2}\right)\right.$

A função hiperbólica pode ser truncada à direita, em função do diâmetro máximo do povoamento, sendo descrita da seguinte forma:

$$
f(x)=\left\{\frac{\frac{\gamma}{\beta}\left(\frac{x-\alpha}{\beta}\right)^{(\gamma-1)}\left(1-\tanh \left(\left(\frac{x-\alpha}{\beta}\right)^{\gamma}\right)^{2}\right)}{\tanh \left(\left(\frac{T-\alpha}{\beta}\right)^{\gamma}\right)}\right.
$$

em que $T$ é o diâmetro máximo da parcela. Para realização dos ajustes, os dados de distribuição diamétrica observada em cada parcela em todas as medições foram agrupados em classes com amplitude do 1,0 cm. Testou-se a aderência das funções aos dados pelo teste de Kolmogorov-Smirnorv (KS) no nível de $1 \%$ de significância (GIBBONS; SUBHABRATA, 1992). 


\subsection{Modelos de distribuição diamétrica}

A redistribuição teórica dos diâmetros por classe foi realizada por meio da alternativa tradicional de correlacionar parâmetros da função com características do povoamento em idades atuais ou futuras, conforme feito por Nogueira et al. (2005). Consideraram-se como variáveis dependentes os parâmetros da função hiperbólica nas quatro formas ajustadas em uma idade futura e como variáveis independentes, os parâmetros dessa mesma função em uma idade atual e as características do povoamento nas idades atual e futura. Os seguintes atributos do povoamento foram utilizados: idade, diâmetro máximo, diâmetro mínimo, diâmetro quadrático e número de árvores. Os ajustes das equações foram efetuados no programa Statistica 8.0 (STATSOFT, INC 2010).

\subsection{Avaliação da redistribuição teórica}

A redistribuição teórica dos diâmetros foi avaliada pela capacidade do sistema de equações em projetar o diâmetro máximo, o número total de árvores e os parâmetros da função hiperbólica ( $\alpha, \beta$ e $\gamma$ ) para qualquer idade. O coeficiente de correlação entre os valores observados e estimados de frequência por classe de diâmetro e a análise gráfica dos resíduos foram utilizados para avaliar a exatidão das estimativas de frequência por classe de diâmetro. Foram avaliadas a consistência, a capacidade de projeção e a realidade biológica do sistema de equações, projetando diferentes distribuições iniciais observadas para idades futuras e comparando com os respectivos valores verificados de frequência por classe de diâmetro. Para avaliar a exatidão das estimativas geradas pelas equações que compõem o modelo de distribuição de diâmetros foram estimadas, ainda, as seguintes estatísticas: raiz quadrada do erro médio (RMSE), bias e bias\% (SIIPILEHTO, 2000). Os estimadores utilizados foram:

$$
\begin{gathered}
R M S E=\sqrt{\frac{\sum_{i=1}^{n}\left(Y_{i}-\hat{Y}_{i}\right)^{2}}{n-1}} \\
\text { bias }=\frac{1}{n} \sum_{i=1}^{n}\left(Y_{i}-\hat{Y}_{i}\right) \\
\text { bias }_{\%}=100 \frac{1}{n} \sum_{i=1}^{n}\left[\left(Y_{i}-\hat{Y}_{i}\right) / Y_{i}\right] \text { Eq. }
\end{gathered}
$$

em que e $Y_{i}$ e $\hat{Y}_{i}=$ valores observados e estimados da variável sob análise; e $n=$ número de casos.

\section{RESULTADOS}

As frequências estimadas de cada parcela e idade foram comparadas com as observadas. Todos os ajustes apresentaram aderência aos dados pelo teste Kolmogorov-Smirnorv ( $\mathrm{p}>0,01)$.

A análise gráfica dos resíduos utilizada para escolha das equações para compor os modelos de distribuição diamétrica é apresentada na Figura 1. As equações que compõem os modelos ajustados são apresentadas a seguir:

$$
\begin{gathered}
d \min _{2}=\operatorname{dmin}_{1} e^{\left(-(-1355,7520)\left(I_{2}^{0,0002}-I_{1}^{0,0002}\right)\right.} R_{Y \hat{Y}}=0,9245 \\
\operatorname{dmax}_{2}=\operatorname{dmax}_{1} e^{\left(-(-1,0015)\left(I_{2}^{0,1722}-I_{1}^{0,1722}\right)\right.} R_{Y \hat{Y}}=0,9786 \\
N_{2}=N_{1} e^{\left(-(195,9842)\left(I_{2}^{-0,0004}-I_{1}^{-0,0004}\right)\right.} \quad R_{Y \hat{Y}}=0,8444
\end{gathered}
$$

\section{Função hiperbólica com três parâmetros}

$$
\begin{gathered}
\operatorname{Ln} \gamma_{2}=0,2390 e^{\left(-(22,1737)\left(I_{2}^{-0.3215}-I_{1}^{-0.3215}\right)\right.}+0,1045 \beta_{2}+0,0636 \operatorname{Ln} \gamma_{1} \quad R_{Y \hat{Y}}=0,9538 \\
\operatorname{Ln} \beta_{2}=\operatorname{Ln} \beta_{1}\left(\frac{I_{1}}{I_{2}}\right)+0,1776\left(1-\frac{I_{1}}{I_{2}}\right) d \max _{2} \quad R_{Y \hat{Y}}=0,7085 \\
\alpha_{2}=\alpha_{1} e^{\left(-(1,0154)\left(I_{2}^{0,2847}-I_{1}^{0,2847}\right)\right.} \quad R_{Y \hat{Y}}=0,8300
\end{gathered}
$$

Função hiperbólica com dois parâmetros

$$
\begin{gathered}
\operatorname{Ln} \gamma_{2}=\operatorname{Ln} \gamma_{1} e^{\left(-(-2473,8706)\left(I_{2}^{0,0001}-I_{1}^{0,0001}\right)\right.} \quad R_{Y \hat{Y}}=0,7536 \\
\beta_{2}=\beta_{1}\left(\frac{I_{1}}{I_{2}}\right)+1,2599\left(1-\frac{I_{1}}{I_{2}}\right) d \max _{1} \quad R_{Y \hat{Y}}=0,9937
\end{gathered}
$$

\section{Função hiperbólica $\operatorname{com} \alpha=\operatorname{dmin}$}

$$
\begin{array}{ll}
\operatorname{Ln} \gamma_{2}=\operatorname{Ln} \gamma_{1}\left(\frac{I_{1}}{I_{2}}\right)+1,3876\left(1-\frac{I_{1}}{I_{2}}\right) & R_{Y \hat{Y}}=0,7656 \\
\operatorname{Ln} \beta_{2}=\operatorname{Ln} \beta_{1}\left(\frac{I_{1}}{I_{2}}\right)+2,2795\left(1-\frac{I_{1}}{I_{2}}\right) & R_{Y \hat{Y}}=0,7138
\end{array}
$$



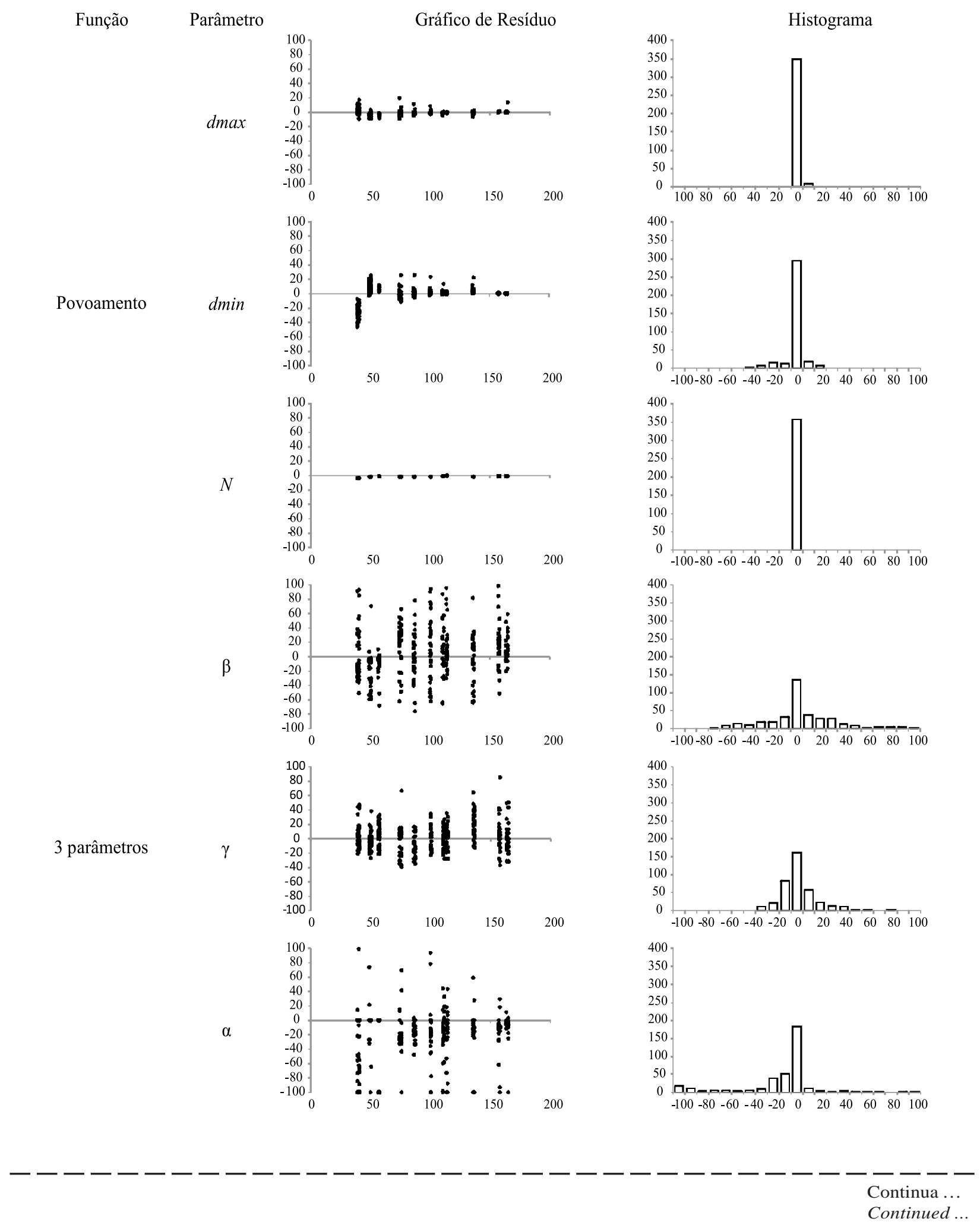

Revista Árvore, Viçosa-MG, v.35, n.5, p.1069-1076, 2011

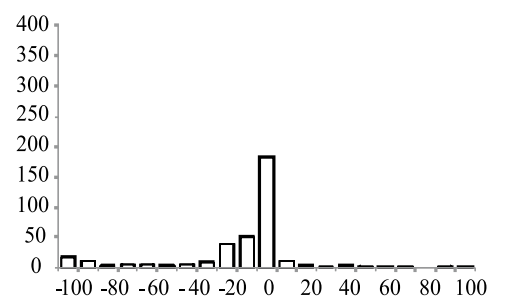


Figura 1 - Cont.

Figure 1-Cont.

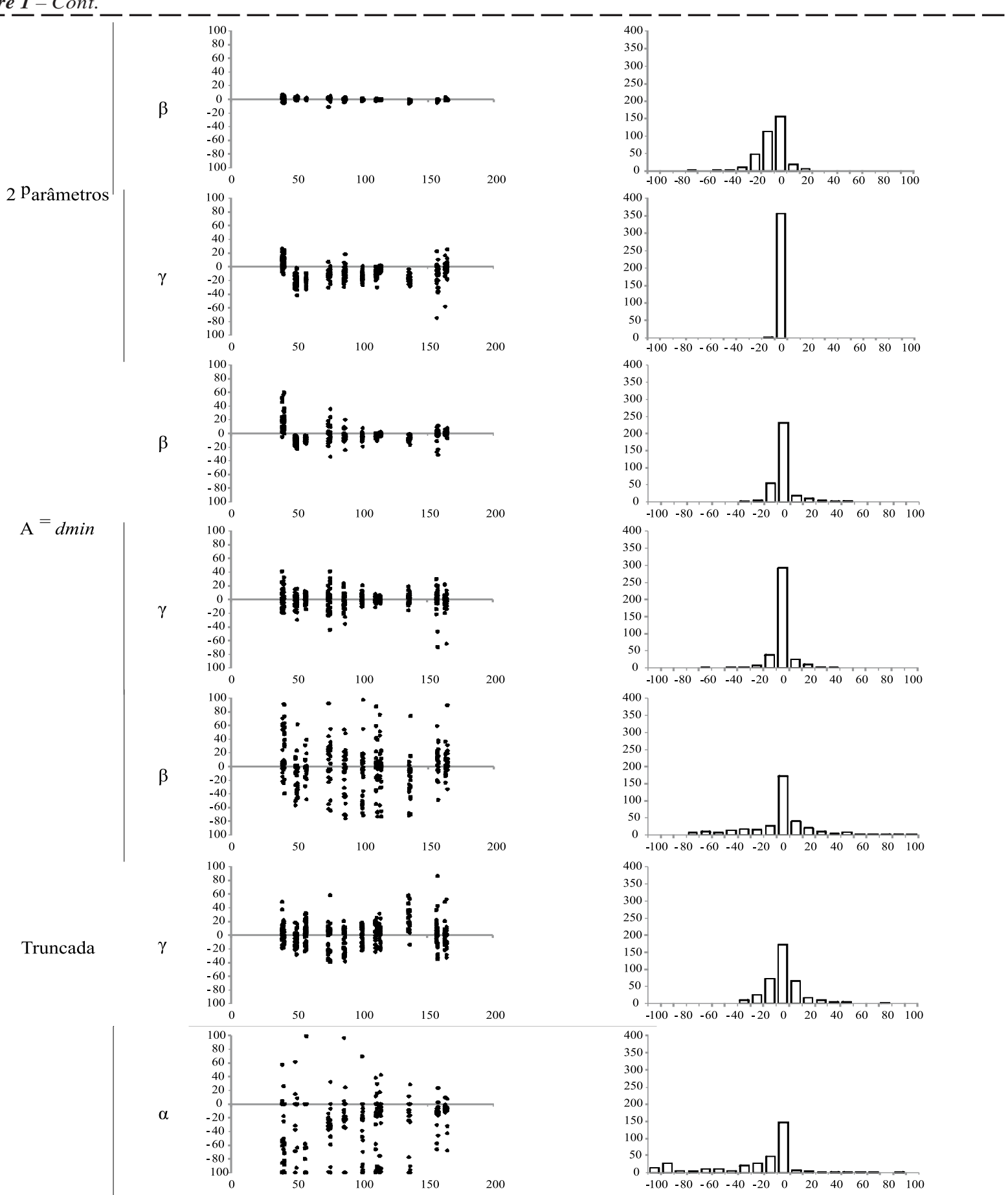

Figura 1 - Gráfico de resíduos e histograma de classes de erro para as equações obtidas da projeção da distribuição diamétrica com diferentes formas da função hiperbólica.

Figure 1 - Graph of the residues and histogram of error classes for equations obtained from projection of diameter distribution with different forms of the hyperbolic function. 


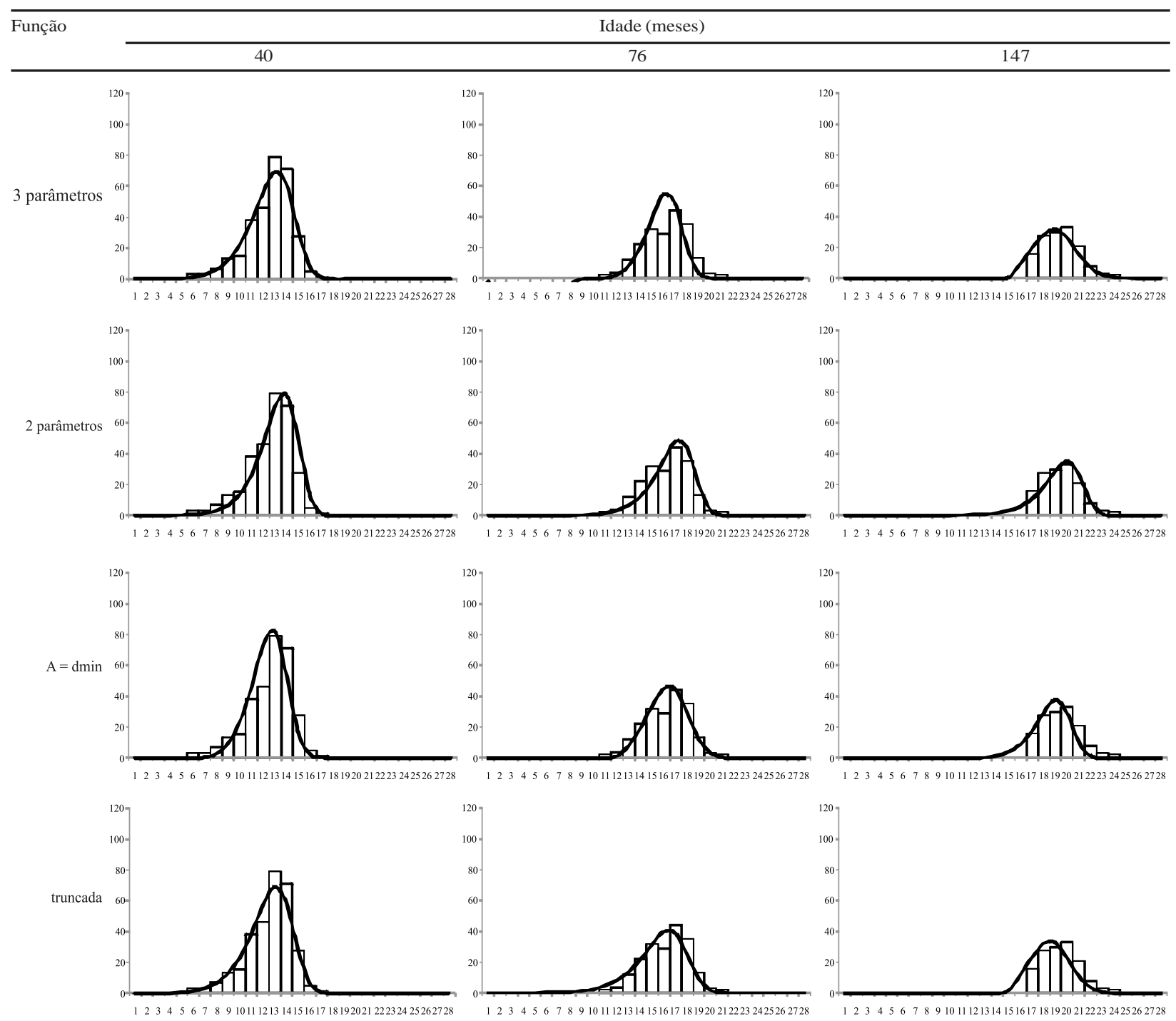

Figura 2 - Projeções da distribuição diamétrica para uma parcela escolhida ao acaso para os modelos propostos, com as quatro formas da função hiperbólica.

Figure 2 - Projections of the diameter distribution for a randomly chosen plot forproposed models, with the four forms of the hyperbolic function.

\section{Função hiperbólica truncada}

$$
\begin{gathered}
L n \gamma_{2}=0,2552 e^{\left(-(28,0959)\left(I_{2}^{-0,5446}-I_{1}^{-0.456}\right)\right.}+0,1037 \beta_{2}+0,0593 \operatorname{Ln\gamma _{1}} \quad R_{Y \hat{Y}}=0,9602 \\
\operatorname{Ln} \beta_{2}=\operatorname{Ln} \beta_{1}\left(\frac{I_{1}}{I_{2}}\right)+3,0122\left(1-\frac{I_{1}}{I_{2}}\right) \quad R_{Y \hat{Y}}=0,6929 \\
\alpha_{2}=\alpha_{1} e^{\left(-(1,8971)\left(I_{2}^{0,2409}-I_{1}^{0,2409}\right)\right.} \quad R_{Y \hat{Y}}=0,7898
\end{gathered}
$$

em que $I_{1}$ e $I_{2}$ são as idades atual e futura, respectivamente, em meses; $\gamma_{1}$ e $\gamma_{2}$ são os parâmetros de forma função hiperbólica nas idades atual e futura; $\beta_{1}$ e $\beta_{2}$ são os parâmetros de escala nas idades atual e futura; $\alpha_{1}$ e $\alpha_{2}$ são os parâmetros de escala nas idades atual e futura; $d \max _{1}$ e $\operatorname{dmax}_{2}$ são os diâmetros máximos nas idades atual e futura, em cm; $d \min _{1}$ e $d \min _{2}$ são os diâmetros mínimos nas idades atual e futura, em

Revista Árvore, Viçosa-MG, v.35, n.5, p.1069-1076, 2011 
cm; $N_{1}$ e $N_{2}$ são as frequências de árvores por hectare nas idades atual e futura; e $L n$ indica o logaritmo neperiano.

Na Figura 2, verifica-se a evolução da distribuição diamétrica realizada em uma parcela escolhida ao acaso em três das idades mensuradas com os quatro modelos propostos, com intensidade de desbaste de 35\%. Os valores de RMSE, bias e bias\% dos modelos ajustados são apresentados na Tabela 1.

\section{DISCUSSÃO}

Este estudo é o primeiro a comparar a performance do ajuste de diferentes formas da função hiperbólica para a projeção da distribuição diamétrica de povoamentos desbastados de eucalipto. Os dados utilizados descrevem bem as variações encontradas em plantios clonais de eucalipto nas condições edafoclimáticas descritas, sendo o número de parcelas e medições utilizadas representativos em estudos de modelagem (CAMPOS; LEITE, 2009).

A aderência aos dados pela função hiperbólica foi comprovada pelo teste KS, o que demonstra a flexibilidade da função na descrição da estrutura diamétrica de povoamentos desbastados. Diversos autores utilizam esses critérios para a escolha da $f d p$ a ser utilizada (BARRA at al., 2004; LEITE et al., 2010).

A utilização do parâmetro de locação para o ajuste das funções acarreta diminuição significativa nos coeficientes de correlação entre valores observados

Tabela 1 - Valores de RMSE, bias e bias\% das equações ajustadas para as diferentes formas de utilização da função hiperbólica.

Table 1 - Values of RMSE, bias and bias\% of equations adjusted for the different ways of using hyperbolic function.

\begin{tabular}{lcccc}
\hline Função & Parâmetro & RMSE & bias & bias\% \\
\hline Povoamento & $d$ min & 1,6977 & 0,1054 & $-0,3786$ \\
& $d \max$ & 2,5175 & 2,0430 & $-10,9901$ \\
& $N$ & 56,1188 & $-1,0759$ & $-6,6787$ \\
\hline \multirow{2}{*}{ parâmetros } & $\beta$ & 4,0328 & 0,1383 & $-12,1275$ \\
& $\gamma$ & 1,0751 & 0,0326 & $-1,6113$ \\
\hline \multirow{2}{*}{ parâmetros } & $\beta$ & 3,8660 & 0,4745 & $-44674,0971$ \\
\hline$\alpha=$ dmin & $\beta$ & 0,3739 & $-0,0173$ & 0,2908 \\
& $\gamma$ & 1,9393 & 0,4712 & $-5,4941$ \\
\hline \multirow{2}{*}{ Truncada } & $\gamma$ & 0,7492 & 0,0036 & $-3,6825$ \\
\hline
\end{tabular}

e estimados das equações que compõem os modelos de crescimento e produção. Em função da aplicação dos desbastes, a recuperação do parâmetro de locação fica comprometida, o que pode ser observado pelo valor de bias\% obtidos nas equações desse parâmetro, sugerindo a subestimação do valor deste (Tabela 1). Contudo, essa variação pode ser explicada pelo fato de o valor do parâmetro em determinadas situações convergir para a origem em distribuições mais achatadas, ou para o valor do diâmetro mínimo em distribuições mais estreitas.

Apesar da alta dispersão dos resíduos para as funções com a utilização do parâmetro de locação, a análise do histograma de resíduos demonstra a não existência de tendenciosidade na recuperação dos parâmetros em idades futuras.

\section{CONCLUSÃO}

Conclui-se que a função hiperbólica nas formas testadas pode ser utilizada para a descrição e projeção da estrutura diamétrica de povoamentos de eucalipto submetidos a desbastes, podendo a não supressão do parâmetro de locação apresentar redução na eficiência das estimativas dos modelos.

\section{REFERÊNCIAS}

BARRA, O. S. V. et al. Proposta metodológica para o ajuste ótimo da distribuição diamétrica Weibull 3P. Floresta, v.34, n.3, p.387-393, 2004.

BURKHART, H. E.; CAO, Q. V.; WARE, Z. D. A. A comparison of growth and yield prediction models for loblolly pine. Blacksburg: Virginia Polytechnic Institute and State University, Scholl of Forestry and Wildlife Resources, 1981. 59p

CAMPOS, J. C. C.; LEITE, H. G. Mensuração florestal: Perguntas e respostas. 3.ed. Viçosa, MG: Universidade Federal de Viçosa, 2009. 548p.

CLUTTER, J. L. et al. Timber management: a quantitative approach. New York: John Willey \& Sons, 1983. 333p.

DEMOLINARI, R. A. et al. Crescimento de plantios clonais de eucalipto não desbastados na região de Monte Dourado (PA). Revista Árvore, v.31, n.3, p.503-512, 2007.

GIBBONS, J. D.; SUBHABRATA, C. Nonparametric statistical inference. 3.ed. New York: Marcel Dekker, 1992. 544p. (Statistics: textbook and monograph, 31). 
GUIMARÃES, D. P. Desenvolvimento de um modelo de distribuição

diamétrica de passo invariante para prognose e projeção da estrutura de povoamentos de eucalipto. 178f. Tese (Doutorado em Ciência Florestal) -

Universidade Federal de Viçosa, Viçosa, MG, 1994.

GUIMARÃES, D. P. Uma função

hiperbólica de distribuição

probabilística de alta flexibilidade.

Planaltina: Embrapa Cerrados, 2002. 40p.
LEITE, H. G. et al. Avaliação do ajuste das funções Weibull e hiperbólica a dados de povoamentos de eucalipto submetidos a desbaste. Revista Árvore, v.34, n.2, p.305-311, 2010.

NOGUEIRA, G. S. et al. Modelo de distribuição diamétrica para povoamentos de Eucalyptus sp. submetidos a desbaste. Revista Árvore, v.29, n.4, p.579-589, 2005.

SIIPILEHTO, J. A comparison of two parameter prediction methods for stand structure in Finland. Silva Fennica, v.34, n.4, p.331-349, 2000 